\title{
A SHORT PROOF OF AN IDENTITY OF SYLVESTER
}

\section{GAURAV BHATNAGAR}

(Received 16 October 1996)

\begin{abstract}
We present two short proofs of an identity found by Sylvester and rediscovered by Louck. The first proof is an elementary version of Knuth's proof and is analogous to Macdonald's proof of a related identity of Milne. The second is Sylvester's own proof of his identity.

Keywords and phrases. Sylvester's identity, Louck's identity, Milne's identity, complete homogeneous symmetric function, multiple basic hypergeometric series.
\end{abstract}

1991 Mathematics Subject Classification. Primary 01A55, 05E05; Secondary 33D70.

1. Sylvester's identity. Our purpose in this paper is to present two proofs of a fundamental identity found in Sylvester's work, which is in Sylvester's own words [17, p. 90], "a simple theorem for expressing, by means of partial fractions, the sum of the homogeneous powers and products of any number of quantities."

The identity in question is

$$
h_{q-n+1}(\mathbf{x})=\sum_{r=1}^{n} x_{r}^{q} \prod_{\substack{i=1 \\ i \neq r}}^{n} \frac{1}{\left(x_{r}-x_{i}\right)},
$$

where $q$ is a nonnegative integer and the complete homogeneous symmetric function $h_{m}(\mathbf{x})$ in the variables $\mathbf{x} \equiv\left(x_{1}, \ldots, x_{n}\right)$ is defined by means of the generating function

$$
\sum_{m \geq 0} h_{m}(\mathbf{x}) t^{m}=\prod_{i=1}^{n} \frac{1}{1-x_{i} t} .
$$

Further, if $m<0$, then $h_{m}(\mathbf{x})$ is defined to be 0 .

Sylvester [16, p. 42] uses the fact that the sum in (1.1) is 0 when $q=0, \ldots, n-2$, and is a polynomial when $q \geq n-1$. In his later work on partitions [17], he uses (1.1) again. But the identity is most clearly formulated only in the lectures he gave in 1859 [18, p. 156]. A little more than a hundred years later, (1.1) was rediscovered by Louck [8]. Chen and Louck [2] have pointed out that for $q=0,1, \ldots, n-1$, the identity was known to Waring [20] in 1779. The $q=n-1$ case of (1.1) was rediscovered by Good [4] in his elegant proof of Dyson's [3] conjecture.

In Section 2, we will present two short proofs of Sylvester's theorem. Both involve partial fraction expansions. The first proof succeeds in finding the left hand side of (1.1) by starting from the sum in the right hand side and is analogous to Macdonald's proof of a related identity. The second is Sylvester's own proof of his identity and, as suggested by his description above, transforms the left hand side of (1.1) into the 
sum on the right. Finally, in Section 3, we comment briefly upon the importance of these identities.

2. Partial fractions. We first consider Macdonald's clever proof of an identity found by Milne [12]:

$$
\sum_{r=1}^{n}\left(1-y_{r}\right) \prod_{\substack{i=1 \\ i \neq r}}^{n}\left[\frac{1-x_{i} y_{i} / x_{r}}{1-x_{i} / x_{r}}\right]=1-y_{1} y_{2} \cdots y_{n}
$$

Macdonald (see [13]) proved (2.1) by setting $t=0$ in the partial fraction expansion

$$
\prod_{i=1}^{n} \frac{\left(1-t x_{i} y_{i}\right)}{\left(1-t x_{i}\right)}=y_{1} \cdots y_{n}+\sum_{r=1}^{n} \frac{1-y_{r}}{1-t x_{r}} \prod_{\substack{i=1 \\ i \neq r}}^{n}\left[\frac{1-x_{i} y_{i} / x_{r}}{1-x_{i} / x_{r}}\right] .
$$

Our first proof of Sylvester's identity is analogous to Macdonald's proof of (2.1). To prove (1.1), we consider the partial fraction expansion

$$
z^{q} \prod_{i=1}^{n} \frac{1}{\left(z-x_{i}\right)}=\sum_{r=1}^{n} \frac{x_{r}^{q}}{z-x_{r}} \prod_{\substack{i=1 \\ i \neq r}}^{n} \frac{1}{\left(x_{r}-x_{i}\right)}+p_{q}(z),
$$

where $p_{q}(z)=0$ if $q=0,1, \ldots, n-1$. Further, if $q \geq n$, then it is a polynomial of degree $q-n$. Let $F_{q}\left(x_{1}, \ldots, x_{n}\right)$ represent the sum on the right hand side of (1.1). Next, set $z=0$ in (2.3) to obtain

$$
F_{q-1}\left(x_{1}, \ldots, x_{n}\right)=p_{q}(0) .
$$

Our proof will be complete once we compute $p_{q}(0)$. But $p_{q}(0)$ is nothing but the constant term in the quotient obtained when $z^{q}$ is divided by $\prod_{i=1}^{n}\left(z-x_{i}\right)$. That is,

$$
\begin{aligned}
p_{q}(0) & =\text { the constant term in } z^{q} \prod_{i=1}^{n} \frac{1}{\left(z-x_{i}\right)} \\
& =\text { the coefficient of } z^{n-q} \text { in } \prod_{i=1}^{n} \frac{1}{\left(1-x_{i} / z\right)} \\
& =h_{q-n}(\mathbf{x}),
\end{aligned}
$$

by comparing with (1.2), it follows that

$$
F_{q}\left(x_{1}, \ldots, x_{n}\right)=p_{q+1}(0)=h_{q-n+1}(\mathbf{x}) .
$$

This completes the derivation of Sylvester's identity.

Macdonald's proof of (2.1) is very simple, but the choice of the particular rational function on the left hand side of (2.2) is unmotivated. A similar remark holds for (2.3). However, a simple observation remedies this situation.

Once again, consider the sum side of (1.1), where $n$ is replaced by $n+1$, and $x_{n+1}$ is renamed $z$. In this manner, we obtain

$$
F_{q}\left(x_{1}, \ldots, x_{n}, z\right)=\sum_{r=1}^{n} \frac{x_{r}^{q}}{x_{r}-z} \prod_{\substack{i=1 \\ i \neq r}}^{n} \frac{1}{\left(x_{r}-x_{i}\right)}+z^{q} \prod_{i=1}^{n} \frac{1}{\left(z-x_{i}\right)} .
$$

It is clear that (2.7) is the same as (2.3), our starting point in the proof of Sylvester's 
identity. The particular choice of the rational function considered is now transparent. The same observation applies to Macdonald's proof of Milne's identity.

This observation is also relevant to Askey's proof of Milne's identity, which is reproduced by Milne [13]. Askey first proved that the sum side of (2.1) is independent of $x_{1}, \ldots, x_{n}$. Suppressing even the dependence on $y_{1}, \ldots, y_{n}$, we let $f_{n}$ denote the left hand side of (2.1). To complete his proof, Askey found a simple recursion for $f_{n}$ :

$$
f_{n+1}=y_{n+1} f_{n}+\left(1-y_{n+1}\right),
$$

from which (2.1) follows quite easily.

Instead, we find another recursion for $f_{n}$ by replacing $n$ by $n+1$ in (2.1) and taking the limit as $x_{n+1} \rightarrow 0$. In this manner, we obtain

$$
f_{n+1}-f_{n}=y_{1} \cdots y_{n}-y_{1} \cdots y_{n+1} .
$$

We also have the initial condition $f_{1}=1-y_{1}$. Milne's identity follows by noting that

$$
\begin{aligned}
f_{1}+\sum_{r=1}^{n-1}\left(f_{r+1}-f_{r}\right) & =1-y_{1}+\sum_{r=1}^{n-1}\left(y_{1} \cdots y_{r}-y_{1} \cdots y_{r+1}\right) \\
& =1-y_{1} \cdots y_{n},
\end{aligned}
$$

by telescoping. Recursion (2.9) is perhaps even simpler than Askey's recursion.

The proof of (1.1) presented above is also related to Sylvester's proof of his identity. In Sylvester's notes [18], where (1.1) appears explicitly, he does not include his proof. But based on his remarks reproduced above and some of his work in his previous paper [17], it seems likely that he obtained (1.1) by considering the partial fraction expansion

$$
\prod_{i=1}^{n} \frac{1}{z-x_{i}}=\sum_{r=1}^{n} \frac{1}{z-x_{r}} \prod_{\substack{i=1 \\ i \neq r}}^{n} \frac{1}{\left(x_{r}-x_{i}\right)} .
$$

By equating the coefficients of $z^{-q-1}$ on both sides of the equation, we immediately obtain (1.1). Compare this with our computation of $p_{q}(0)$ above.

It is interesting to note that setting $z=0$ in (2.11) and replacing $x_{i}$ by $x_{i}^{-1}$, we obtain Good's identity, the $q=n-1$ case of (1.1).

3. Concluding remarks. Our first proof of Sylvester's identity is an elementary version of the proof given by Knuth [7, §1.2.3, problem 33], who found it necessary to use Cauchy's residue theorem. Variations of Sylvester's proof are given by Chen and Louck [2] and Strehl and Wilf [15], though these authors prefer to use the Lagrange interpolation formula rather than partial fractions. Knuth mentions that special cases of (1.1) are useful in the theory of divided differences. Indeed, (1.1) has been rediscovered by Verde-Star [19] in this context. It appears in the context of mathematical physics in the work of Louck and Biedenharn [9, 10]. Far reaching generalizations of (1.1) have been found by Gustafson and Milne [6] and by Chen and Louck [2].

Milne [12] first proved (2.1) using (1.1). Several other proofs of (2.1), including those of Macdonald and Askey, are compiled by Milne [13]. Yet another proof is given by 
Strehl and Wilf [15]. Identity (2.1) is fundamental in the study of multiple basic hypergeometric series. See, for instance, Milne [11, 13] and Gustafson [5].

Finally, we note that Macdonald's proof of (2.1) is also relevant. Bhatnagar and Milne [1] and Schlosser [14] have used (2.2) to generalize the identities which Milne [11] found using (2.1).

ACKNOWLEDGEMENTS. We thank Steve Milne for passing on A. Lascoux's remark that Sylvester's work is peppered with the kind of identities which appear in this paper. It is likely that Milne's identity is also classical. It would be interesting to know about its history. We also thank Mary Scott, Ohio State University's mathematics librarian, for finding the correct reference for Waring [20].

\section{REFERENCES}

[1] G. S. Bhatnagar and S. C. Milne, Generalized bibasic hypergeometric series and their $U(n)$ extensions, Adv. in Math. 131 (1997), no. 1, 188-252. Zbl 885.33011.

[2] W. Y. C. Chen and J. D. Louck, Interpolation for symmetric functions, Adv. in Math. 117 (1996), no. 1, 147-156. MR 97g:05165. Zbl 857.05092.

[3] F. J. Dyson, Statistical theory of the energy levels of complex systems. I, J. Mathematical Phys. 3 (1962), 140-156. MR 26\#1111. Zbl 105.41604.

[4] I. J. Good, Short proof of a conjecture by Dyson, J. Mathematical Phys. 11 (1970), 1884. MR 41\#3290.

[5] R. A. Gustafson, The Macdonald identities for affine root systems of classical type and hypergeometric series very-well-poised on semisimple Lie algebras, Ramanujan International Symposium on Analysis (Pune, 1987) (New Delhi), Macmillan of India, 1989, pp. 185-224. MR 92k:33015.

[6] R. A. Gustafson and S. C. Milne, Schur functions, Good's identity, and hypergeometric series well poised in SU(n), Adv. in Math. 48 (1983), no. 2, 177-188. MR 84m:05013. Zbl 516.33015.

[7] D. E. Knuth, The art of computer programming vol 1: Fundamental algorithms, second printing of the 2nd ed., vol. XXII, Addison-Wesley Series in Computer Science and Information Processing, no. 634, Addison-Wesley Publishing Co., London, Amsterdam, 1968. MR 51 14624. Zbl 191.17903.

[8] J. D. Louck, Theory of angular momentum in N-dimensional space, Tech. Report LA-2451, Los Alamos Scientific Laboratory, 1960.

[9] J. D. Louck and L. C. Biedenharn, Canonical unit adjoint tensor operators in $U(n)$, J. Mathematical Phys. 11 (1970), 2368-2414. MR 45 6295. Zbl 196.14501.

[10] On the structure of the canonical tensor operators in the unitary groups. III. Further developments of the boson polynomials and their implications, J. Mathematical Phys. 14 (1973), 1336-1357. MR 496807.

[11] S. C. Milne, An elementary proof of the Macdonald identities for $A_{l}^{(1)}$, Adv. in Math. 57 (1985), no. 1, 34-70. MR 87e:17020. Zbl 586.33011.

[12] _ A q-analog of hypergeometric series well-poised in $\mathrm{SU}(n)$ and invariant $G$ functions, Adv. in Math. 58 (1985), no. 1, 1-60. MR 87d:22028. Zbl 586.33014.

[13] , A q-analog of the Gauss summation theorem for hypergeometric series in $\mathrm{U}(n)$, Adv. in Math. 72 (1988), no. 1, 59-131. MR 90c:33006. Zbl 658.33005.

[14] M. Schlosser, Multidimensional matrix inversions and $A_{r}$ and $D_{r}$ basic hypergeometric series, Ramanujan J. 1 (1997), no. 3, 243-274. Zbl 980.45703.

[15] V. Strehl and H. S. Wilf, Five surprisingly simple complexities, J. Symbolic Comput. 20 (1995), no. 5-6, 725-729. MR 97j:05011. Zbl 851.68053.

[16] J. J. Sylvester, On rational derivation from equations of coexistence, that is to say, a new and extended theory of elimination, Part I, Philos. Mag. 15 (1839), 428-435, 
reprinted in Collected Mathematical Papers I 40-46 reprinted by Chelsea; New york, 1973.

[17] _ On the partition of numbers, Quart. J. Math. 1 (1857), 141-152, reprinted in Collected Mathematical Papers II 90-99 reprinted by Chelsea; New york, 1973.

[18] _ Outlines of seven lectures on the partition of numbers, Proc. Lond. Math. Soc. 28 (1897), 33-96, reprinted in Collected Mathematical Papers II 119-175 reprinted by Chelsea; New york, 1973.

[19] L. Verde-Star, Divided differences and combinatorial identities, Stud. Appl. Math. 85 (1991), no. 3, 215-242. MR 92i:65027. Zbl 776.65008.

[20] E. Waring, Problems concerning interpolations, Philos. Trans. Roy. Soc. 69 (1779), 59-67.

Bahatnagar: DePartment of MATHEMATICs,The OHio State University, Columbus, OH 43210, USA

E-mail address: gaurav@math . ohio-state.edu 


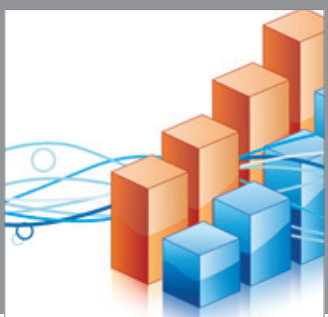

Advances in

Operations Research

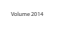

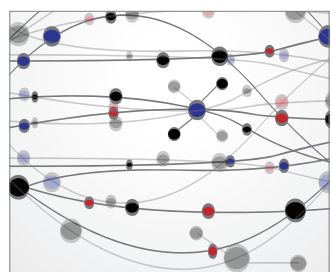

\section{The Scientific} World Journal
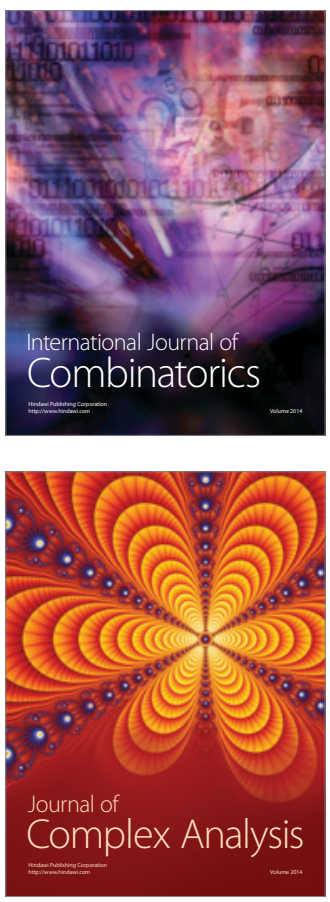

International Journal of

Mathematics and

Mathematical

Sciences
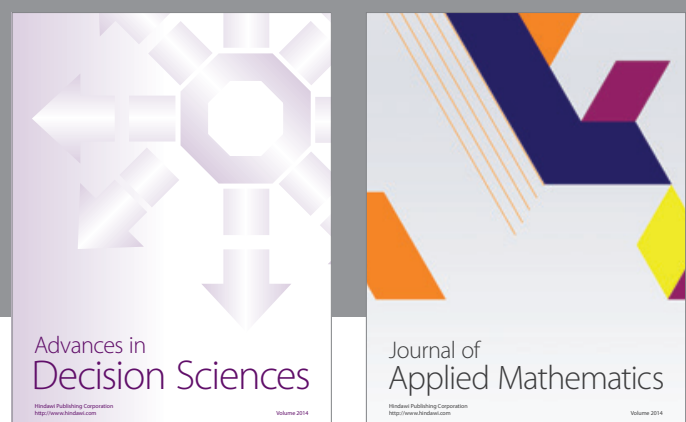

Journal of

Applied Mathematics
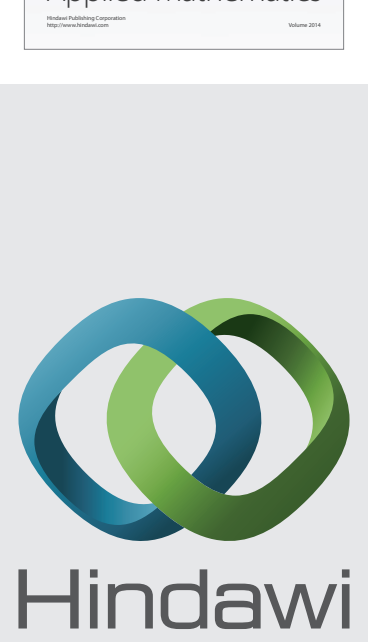

Submit your manuscripts at http://www.hindawi.com
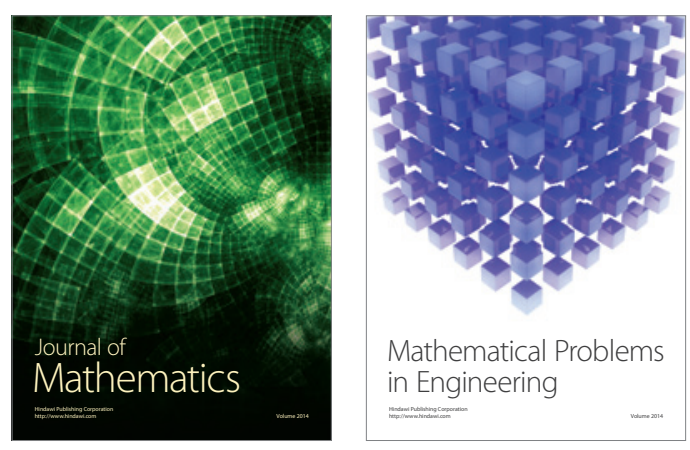

Mathematical Problems in Engineering
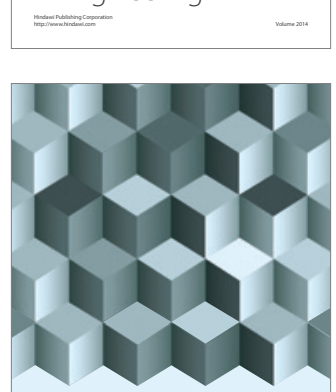

Journal of

Function Spaces
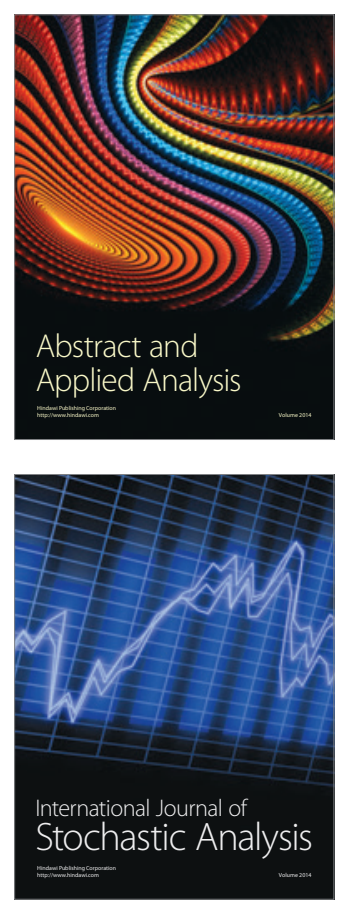

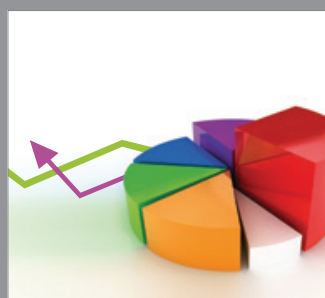

ournal of

Probability and Statistics

Promensencen
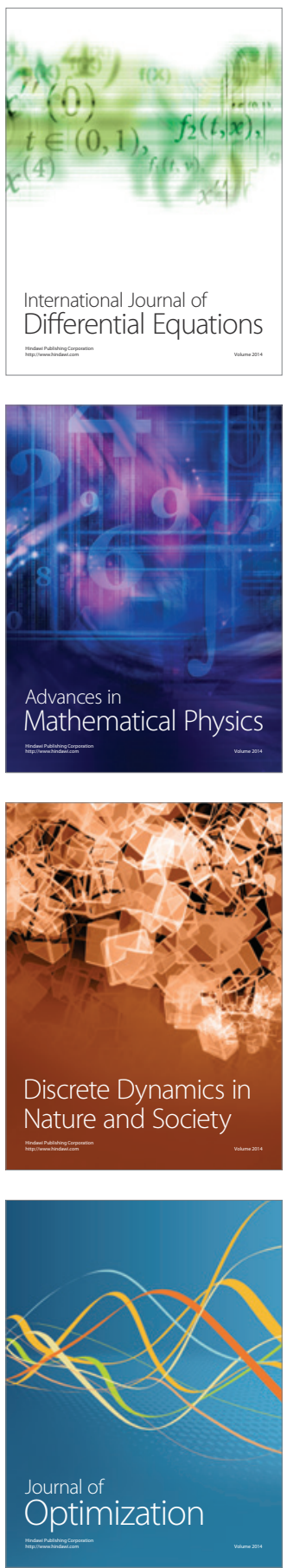